\title{
Transcriptome response to heat stress in a chicken hepatocellular carcinoma cell line
}

\author{
Liang Sun ${ }^{1} \cdot$ Susan J. Lamont ${ }^{2} \cdot$ Amanda M. Cooksey $^{3} \cdot$ Fiona McCarthy $^{3}$ • \\ Catalina O. Tudor ${ }^{4} \cdot$ K. Vijay-Shanker ${ }^{4} \cdot$ Rachael M. DeRita $^{1} \cdot$ Max Rothschild $^{2}$ • \\ Chris Ashwell $^{5} \cdot$ Michael E. Persia $^{2} \cdot$ Carl J. Schmidt $^{1}$ (D)
}

Received: 8 March 2015 /Revised: 22 June 2015 / Accepted: 30 June 2015 / Published online: 5 August 2015

(C) The Author(s) 2015. This article is published with open access at Springerlink.com

\begin{abstract}
Heat stress triggers an evolutionarily conserved set of responses in cells. The transcriptome responds to hyperthermia by altering expression of genes to adapt the cell or organism to survive the heat challenge. RNA-seq technology allows rapid identification of environmentally responsive genes on a large scale. In this study, we have used RNA-seq to identify heat stress responsive genes in the chicken male white leghorn hepatocellular (LMH) cell line. The transcripts of 812 genes were responsive to heat stress $(p<0.01)$ with 235 genes upregulated and 577 downregulated following $2.5 \mathrm{~h}$ of heat stress. Among the upregulated were genes whose products function as chaperones, along with genes affecting collagen synthesis and deposition, transcription factors, chromatin remodelers, and genes modulating the WNT and TGF-beta pathways. Predominant among the downregulated genes were ones that affect DNA replication and repair along with chromosomal segregation. Many of the genes identified in this
\end{abstract}

Electronic supplementary material The online version of this article (doi:10.1007/s12192-015-0621-0) contains supplementary material, which is available to authorized users.

Carl J. Schmidt

schmidtc@udel.edu

1 Department of Animal and Food Sciences, University of Delaware, Newark, DE 19716, USA

2 Department of Animal Science, Iowa State University, Ames, IA 50011, USA

3 School of Animal and Comparative Biomedical Sciences, University of Arizona, Tucson, AZ 85721, USA

4 Department of Computer and Information Sciences, University of Delaware, Newark, DE 19716, USA

5 Department of Poultry Science, North Carolina State University, Raleigh, NC 27695, USA study have not been previously implicated in the heat stress response. These data extend our understanding of the transcriptome response to heat stress with many of the identified biological processes and pathways likely to function in adapting cells and organisms to hyperthermic stress. Furthermore, this study should provide important insight to future efforts attempting to improve species abilities to withstand heat stress through genome-wide association studies and breeding.

Keywords Transcriptome $\cdot$ RNA-seq $\cdot$ Heat stress · Chicken $\cdot$ LMH cells

\section{Introduction}

Fluctuations in environmental temperatures are encountered over the life span of most organisms. Many species have a metabolism that is adapted to the temperature range of the environment in which they evolved. When the external temperature rises above this range, the heat produced by a homothermic animal exceeds the amount that can be lost to the environment, resulting in a rise in body temperature. This triggers an evolutionarily conserved heat stress transcriptome response modulating genes that control multiple cellular activities including protein folding, protein degradation, transport, metabolism, DNA repair, and replication (Lindquist and Craig 1988; Feder and Hofmann 1999; Kregel 2002).

A starting point for understanding the heat stress response is identifying the genes that are modulated by hyperthermia. Numerous studies have identified genes that respond to heat stress, but high-throughput transcriptome sequencing is a technology able to provide a more complete catalog of heat responsive genes (Szustakowski et al. 2007; Li et al. 2011; Islam et al. 2013; Kristiansson et al. 2013; Smith et al. 2013; 
Wang et al. 2013). While the ultimate goal might be to identify every gene in every tissue of an organism that responds to heat stress, a practical first step is to use in vitro cell culture methods. Cultured cells can be raised under defined temperature conditions for precise amounts of time, allowing for careful control of their environment. Subjecting a cell line to heat stress and comparing the transcriptome results with control cells will identify heat responsive genes. The objective of this work was to use transcriptome sequencing to define the heat stress response of the chicken hepatocellular carcinoma cell line, LMH (Kawaguchi et al. 1987), which was developed from a male white leghorn chicken. A total of 812 genes consistently responded to heat stress with 235 induced and 577 repressed following treatment. Enrichment analysis identified functional groupings such as molecular chaperones and transcription factors within the induced genes, and DNA replication and DNA repair among the repressed genes.

\section{Materials and methods}

\section{Cell culture}

LMH cells were obtained from ATCC (Manassas, VA) and cultured in Waymouth's MB medium with $10 \%$ heatinactivated fetal bovine serum in flasks coated with $0.1 \%$ gelatin. Cells were cultured at $37{ }^{\circ} \mathrm{C}$ in $5 \% \mathrm{CO}_{2}$ and passaged every 2-3 days. Prior to heat stress, cells were grown to $80 \%$ confluence. Three T-75 (Falcon) flasks were maintained at control temperature and a second set of three flasks subjected to heat challenge. Six individual flasks of control cells were cultured at $37^{\circ} \mathrm{C}$ while six flasks of heat-stressed cells were maintained at $43{ }^{\circ} \mathrm{C}$ for $2.5 \mathrm{~h}$. This protocol was chosen as prior published work indicated it would provide for maximal heat stress response (Gabis et al. 1996).

\section{RNA preparation}

Cells were released from flasks with trypsin and collected in $50-\mathrm{ml}$ conical tubes by centrifugation (1200 RPM; at $37{ }^{\circ} \mathrm{C}$ for control cells, $43^{\circ} \mathrm{C}$ for heat-stressed cells). RNA was prepared using the Qiagen RNeasy mini kit with DNAase treatment and quality-checked using the Agilent 2100 Bioanalyzer. RNA with a RIN value greater than 9.0 was used for transcriptome library production.

\section{Transcriptome production and data analyses}

Transcriptome libraries were prepared separately from each control and experimental flask using the Illumina TrueSeq RNA Sample Preparation Kit. The 12 individual libraries were sequenced on an Illumina HiSeq 2500 at the University of Delaware DNA Sequencing Facility. Sequences were mapped to the 2006 release of the Red Jungle Fowl genome (International Chicken Genome Sequencing, C 2004) and gene Reads Per Kilobase per Million (RPKM) values assigned using ERANGE (Mortazavi et al. 2008). Expression data were statistically analyzed using JMP, and genes were assigned to functional groups using DAVID (Huang et al. 2009a, 2009b) and eGIFT (Tudor et al. 2010). All fastq files have been submitted to the GEO database under GEO number GSE55321.

\section{Quantitative RT-PCR}

First-strand synthesis was done with superscript II Reverse Transcriptase (Life Technologies) according to the manufacturer's directions. Quantitative reverse transcription PCR (qRT-PCR) was performed using gene-specific primers (Table 2) and the Fast SYBER green master mix (Applied Biosystems) on an Applied Biosystems 7500 Fast Real Time PCR System according to the manufacturer's directions.

\section{Results and discussion}

\section{Overview of results}

Over 250 million transcriptome reads were generated across 12 separate flasks of control or heat-stressed LMH cells (Table 1). A total of 15,945 chicken genes were analyzed in $\mathrm{LMH}$ cells for their response to heat stress at $43{ }^{\circ} \mathrm{C}$

Table 1 Transcriptome Read Depth for Each Sample Library

\begin{tabular}{llll}
\hline Library & Total Reads & Mapped Reads & Unique Reads \\
\hline Control 1 & $26,481,906$ & $89.9 \%$ & $95.9 \%$ \\
Control 2 & $35,224,137$ & $89.8 \%$ & $96.4 \%$ \\
Control 3 & $35,841,457$ & $90.4 \%$ & $95.8 \%$ \\
Control 4 & $22,491,917$ & $87.8 \%$ & $96.0 \%$ \\
Control 5 & $17,147,757$ & $87.3 \%$ & $96.5 \%$ \\
Control 6 & $13,106,307$ & $87.4 \%$ & $96.6 \%$ \\
Heat stress 1 & $33,816,428$ & $90.0 \%$ & $95.2 \%$ \\
Heat stress 2 & $13,710,771$ & $90.0 \%$ & $95.8 \%$ \\
Heat stress 3 & $27,526,906$ & $90.4 \%$ & $96.0 \%$ \\
Heat stress 4 & $19,754,286$ & $87.6 \%$ & $96.2 \%$ \\
Heat stress 5 & $26,463,555$ & $87.7 \%$ & $96.1 \%$ \\
Heat stress 6 & $31,610,408$ & $88.2 \%$ & $96.2 \%$ \\
\hline
\end{tabular}

The total number of sequences from each library prepared from separate flasks of either control $\left(37^{\circ} \mathrm{C}\right)$ or heat stressed $\left(43^{\circ} \mathrm{C}\right) \mathrm{LMH}$ cells is given in the Total Reads column. The Mapped Reads column is the percentage of these total reads that mapped to the chicken genome. The Unique Reads column is the percentage of mapped reads that only correspond to one site in the genome. Combined, this data yielded more than $25 \times 10^{7}$ reads that mapped uniquely to the chicken genome. 
versus $37^{\circ} \mathrm{C}$ for $2.5 \mathrm{~h}$ (Supplementary File 1). A total of 12, 299 genes were detected under heat or control conditions with RPKM values greater than 0.1 . The $\log _{2}$ values of the ratio between the heat stress and control RPKM were determined for each of these genes. A $t$ test was applied to identify genes that were significantly differentially expressed between the two conditions $(p<0.01)$. This generated a list of 812 genes that reproducibly responded to heat challenge across all 12 samples, with 235 genes whose expression was increased and 577 genes whose expression was decreased after heat challenge (Supplementary File 2). Hierarchical clustering (Fig. 1) of the 812 responsive genes segregated the samples based on environmental condition (heat vs. control) with principal component analysis (Supplementary File 3) indicating that $22 \%$ of the variance in the data was associated with the cell incubation temperature. For a complete list of all genes discussed in this paper, see Supplementary File 4.

\section{Validation}

Illumina RNA-seq transcriptome data was validated by quantitative RT-PCR (qRT-PCR) using ten genes, comparing expression levels between control and heat-stressed samples (Table 2). Although the absolute fold changes differed between qRT-PCR and RNA-seq, the direction of change was concordant for each gene. Plotting the qRT-PCR delta $\mathrm{Ct}$ values (difference between heat stress and control $\mathrm{Ct}$ values) versus the $\log _{2}$ RPKM for the genes yielded an $R^{2}$ value of 0.90 (Fig. 2), which is consistent with other studies comparing correlations between RNA-seq and qRT-PCR studies (Core et al. 2008; Nagalakshmi et al. 2008; Camarena et al. 2010; Feng et al. 2010).
Table 2 Gene symbols and primers used for qRT-PCR validation of RNA seq dat

\begin{tabular}{ll}
\hline Gene & Primer $\left(5^{\prime}\right.$-> $\left.{ }^{\prime}\right)$ \\
\hline BAG3_R & GATGGGAGTTGAGGGCTGTA \\
BAG3_F & TACCATCAGGCCCAGAAGAC \\
CAPS2_R & CCCCATGGGTTCCTTAAGAT \\
CAPS2_F & GGCAGGCAAAGCTACAGAAG \\
DNAJA4_R & TCTATTCATTCGGCCTCCAC \\
DNAJA4_F & GAAGTACCACCCCGACAAGA \\
GABRA2_R & TGAATTTCGAGCACTGATGC \\
GABRA2_F & GGCCAAACAATTGGAAAAGA \\
HMOX1_R & GACGCCGTGACCAGCTTGAAC \\
HMOX1_F & GCCACCAAGGAGGTGCACGA \\
HSP25_R & GGCGAAGTTCTTCACATCCT \\
HSP25_F & CACGCAGAGACCATCTTCAG \\
KLHDC2_R & GACGCCTTGTGCCATTATTT \\
KLHDC2_F & GCTCATGCGTGTGCTACAGT \\
MYCN_R & TTGGTTGGATCATGGGTTTT \\
MYCN_F & ACCACTTTTCCATCGGTCAG \\
P4HA2_R & CTGTGATCTGCTGCATTCGT \\
P4HA2_F & AACAACTGGCCAAACCAAAG \\
UCP3_R & GAACGACAAAGGTTGGCAGT \\
UCP3_F & CGGGATTTGATTCTGTGCTT \\
\hline &
\end{tabular}

\section{Heat shock factors}

Four heat shock factors (HSFs), HSF2, HSF3, HSF4, and HSF5, were detectably expressed in the LMH cells (Table 3). HSF2 and HSF3 transcripts were 30-100-fold more abundant than HSF4 and HSF5 although no HSF showed a significant change in expression as a function of temperature (Table 3). In the chicken, HSF1 is not yet mapped to a specific

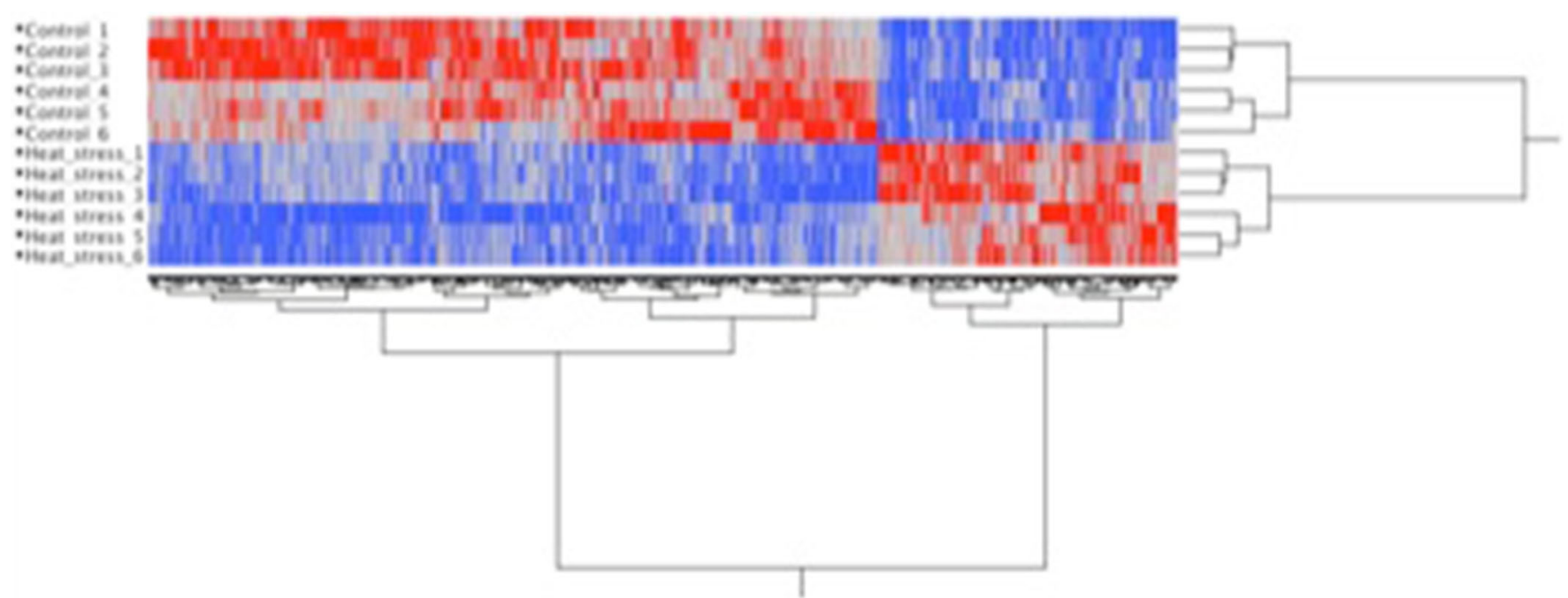

Fig. 1 Hierarchical clustering Hierarchical cluster analysis of transcriptome data from heat challenged and control cells. Note that the control and heat stress samples cluster separately. Red corresponds to genes whose transcripts were enriched in the corresponding sample while blue corresponds to genes whose transcripts were reduced in that sample. 
Fig. 2 Relationship between RNA-seq and qRT-PCR Rsquared analysis of the fold difference relationship between heat challenged and control levels in the expression of 10 genes as determined by transcriptome analysis (X-axis) and quantitative reverse transcriptase PCR (Y-axis).

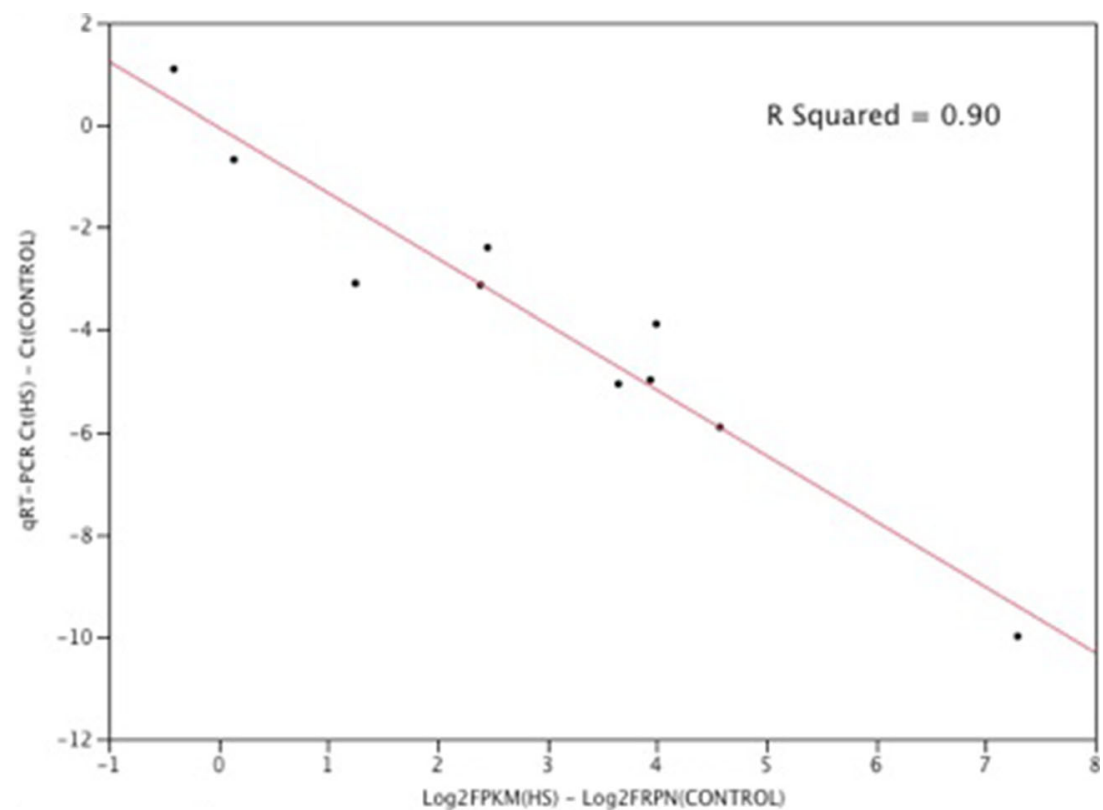

chromosome, and for this reason, we do not have a determined RPKM level for HSF1. However, inspection of the unmapped sequence reads by BLAST identifies chicken HSF1 in the LMH transcriptome. Hence, all five known HSFs are expressed in this cell line.

\section{Chaperones, heat shock protein, and DNAJ genes}

A major function of many heat shock inducible gene products is to serve as protein chaperones, assisting in folding of nascent proteins and inhibiting protein aggregation (Lindquist and Craig 1988; Hightower 1991; Moseley 1997). Fifteen genes encoding chaperones were modulated by heat stress (Table 4). The greatest induction was observed for LOC772158, a heat shock protein 30 (HSP30) like gene product (223-fold), while the least was DNAJB6 (2-fold). Two genes encoding products classified as chaperones, HSPA14 and DNAJC17, exhibited reduced expression following heat treatment.

DAVID functional enrichment analysis (Huang et al. 2009) of gene transcripts elevated by heat stress provided an overview of processes affected by hyperthermia. The functional groupings included Chaperone, Unfolded Protein Binding,

Table 3 RPKM values for Heat Shock Factor (HSF) transcription regulatory genes

\begin{tabular}{lcc}
\hline Heat Shock Factor & RPKM Control & RPKM Heat Stress \\
\hline HSF2 & 46.2 & 32.8 \\
HSF3 & 8.9 & 10.7 \\
HSF4 & 0.1 & 0.1 \\
HSF5 & 0.5 & 0.3 \\
\hline
\end{tabular}

Endoplasmic Reticulum, Protein Kinase Activity, and Regulation of Transcription. eGIFT (Tudor et al. 2010) identifies informative terms (iTerms) for individual genes using text-mining approaches. Because iTerms are not limited to ontology terms, iTerms can provide a finer grained interpretation of gene lists than GO ontology analysis (Table 5). The majority of iTerms refer to processes and functions typically modulated during heat stress including molecular chaperones, co-chaperone, endoplasmic reticulum (ER), secretion, and apoptosis. Many of the gene products in these groups are chaperones that assist in proper protein folding.

Table 4 Fold change for Chaperone gene products

\begin{tabular}{lll}
\hline Entrez Gene ID & Symbol & Fold Change Heat Stress \\
\hline 772158 & LOC772158 & 223 \\
428310 & HSP25 & 157.5 \\
423504 & HSPA2 & 64 \\
416988 & HSPB8 & 36.7 \\
396228 & SERPINH1 & 8.6 \\
418917 & HSPH1 & 8 \\
423463 & HSP90AA1 & 6.5 \\
396487 & HSPA5 & 5.3 \\
395853 & HSPA8 & 4 \\
374163 & HSP90B1 & 3.5 \\
416339 & HSPA4 & 3 \\
427376 & DNAJA1 & 3 \\
420448 & DNAJB6 & 2 \\
770519 & DNAJC17 & 0.4 \\
418802 & HSPA14 & 0.3 \\
\hline
\end{tabular}

Fold change for Chaperone gene products as determined by: (Mean $\operatorname{RPKM}(430 \mathrm{OC}) \div$ Mean $\left.\operatorname{RPKM}\left(37^{\circ} \mathrm{C}\right)\right)$ 
Table 5 iTERMS for gene significantly up-regulated by heat stress

\begin{tabular}{ll}
\hline iTERM & Gene symbols \\
\hline SECRETION & HSP90B1, SFRP1, HSPA5, CYR61, NTNG1, \\
& WNT4, CAPS2, AMH, ABHD5, MYOC, \\
& SERPINH1, CTGF, CBLN1, SYT4, INHBA, \\
& ABCC2 \\
APOPTOSIS & HSPA5, BAG3, HSPA4, LARP6, TIMP3, E2F7, \\
& HSP90AA1, KLF5, HSP25, ATF4, HSPH1, \\
TRAP3K5, TSC22D1, SQSTM1 \\
FACTOR & ATF4, NFYA, RFX2, JDP2, NHLH1, CREM, \\
& PLAG1, TMEM173, NFYA, KLF5, KAT2A, \\
MOLECULAR & SIK1, KLF6, NR0B1, TSC22D1 \\
CHAPERONES & DSP90B1, HSPA5, HSPA2, HSPA4, HSPA8, \\
& HSPHA1, HSP90AA1, SERPINH1, HSP25, \\
STRESS & HSP90B1, HSPA5, BAG3, HSP90AA1, \\
& SERPINH1 HSPA2, HSPA4, \\
& HSPA8, HSP25, ATF4, HSPH1
\end{tabular}

\section{Collagen scaffold}

Six gene products affecting the collagen scaffold were elevated by heat stress: serpin peptidase inhibitor 1 (SERPINH1); La ribonucleoprotein domain family, member 6 (LARP6); TIMP metallopeptidase inhibitor 3 (TIMP3); discoidin domain receptor tyrosine kinase 2 (DDR2); connective tissue growth factor (CTGF); and cysteine-rich, angiogenic inducer, 61 (CYR61). SERPINH1 is a molecular chaperone responsible for proper folding and secretion of collagen (Kurkinen et al. 1984; Nagata et al. 1986; Cates et al. 1987) while LARP6 is a La ribonucleoprotein domain family member that binds the $5^{\prime}$ noncoding region of the collagen mRNA and directs collagen protein synthesis to discrete locations within the endoplasmic reticulum (Cai et al. 2010a, 2010b). TIMP3 (Pavloff et al. 1992) plays a role in the extracellular stabilization of collagen matrices by inhibiting metalloproteases (Leco et al. 2001), while DDR2 functions as a collagen receptor and plays an important role in suppressing liver fibrosis (Olaso et al. 2011). Finally, both CTGF and CYR61 are growth factors that, among other things, promote synthesis and deposition of collagen (Duncan et al. 1999; Brigstock 2002).

\section{Transcription factors}

Several transcription factors were positively regulated by heat stress, two of which, activating transcription factor 4 (ATF4) and nuclear transcription factor A (NFYA), are known to couple ER stress to transcriptional regulation. Also enriched was the transcription factor E2F7 that regulates the cell cycle, with elevated E2F7 expression causing arrest in G2 phase (de Bruin et al. 2003). Fourteen other genes whose products regulate transcription showed a significant increase in expression when LMH cells were heat-stressed (Table 5). Salt-inducible kinase 1 (SIK1) responds to osmotic stress (Wang et al. 1999; Sjostrom et al. 2007), TSC22 domain family, member 1 (TSC22D1) is induced by TGFß (Kester et al. 1999), transmembrane protein 173 (TMEM173) responds to viral infection (Ishikawa et al. 2009), and regulatory factor X (RFX2) is involved in testes development (Horvath et al. 2004; Wolfe et al. 2004), but none of these have previously been shown to be heat responsive. Two members of the Kruppel-like transcription factor family, KLF5 and KLF6, also responded to heat stress in these experiments. To date, only KLF4 has been shown to be induced by heat stress (Liu et al. 2006); in our experiments, KLF4 expression was not detected in LMH cells. Perhaps the paralogous KLF5 and KLF6 gene products replace KLF4 function in LMH cells. Six additional transcription regulatory genes were enriched by heat challenge that have not, to our knowledge, been previously implicated in heat stress response including cAMP responsive element modulator (CREM), v-myc related oncogene, neuroblastoma derived (MYCN), nescient helix loop helix 1 (NHLH1), nuclear receptor subfamily 0 , group $\mathrm{B}$, member 1 (NR0B1), pleiomorphic adenoma gene 1 (PLAG1), and Scm-like with four MBT domains protein 1 (SFMBT1).

\section{Chromatin modification}

Four gene products that were upregulated by heat stress control transcription by chromatin modification. K(lysine) acetyltransferase 2A (KAT2A) promotes histone acetylation (Nagy and Tora 2007), while Jun dimerization protein 2 (JDP2) inhibits (Jin et al. 2006) histone acetylation, jumonji domain containing 6 (JMJD6) is responsible for demethylation of HIS3 at arginine residues (Chang et al. 2007), and SFMBT1 is a member of the polycomb protein family that inhibits transcription by altering chromatin localization (Alfieri et al. 2013). Another four genes controlling epigenetic modulation are downregulated by heat stress: chromatin assembly factor 1, subunit B (CHAF1B); ubiquitin-like with PHD and ring finger domains 1 (UHRF1); DNA (cytosine-5-)-methyltransferase 3 beta (DNMT3B); and enhancer of zeste homolog 2 (EZH2). CHAF1B (Nabatiyan and Krude 2004), UHRF1 (Hashimoto et al. 2009), and DNMT3B (Wang et al. 2007) function in maintaining DNA methylation and silencing genes through incorporation of DNA into heterochromatin. EZH2 is a polycomb family member that is responsible for silencing genes during development by trimethylation of Histone H3 (Rajasekhar and Begemann 2007).

The different transcription factors and chromatin modifiers whose transcripts were affected by heat stress suggest a complex interplay between transcription activators, repressors, and epigenetic modifications in response to heat challenge. For example, KLF5 is a strong transcriptional activator typically expressed in proliferating cells of the gastrointestinal track that plays a role in suppressing apoptosis (Sun et al. 2001). In 
contrast, NR0B1 functions as a transcriptional repressor, possibly affecting the large number of genes whose expression is suppressed by heat stress in the LMH cells. At least eight genes affected by heat stress (up regulated or downregulated) modulate transcription by epigenetic mechanisms and could play an important role in both short- and long-term responses to heat. These epigenetic regulators control different types of modifications including DNA methylation, histone acetylation, and histone methylation. Exposure of chicken embryos to elevated temperatures during embryonic development improves the ability of the hatched bird to tolerate heat stress (Wang and Edens 1998; Pavani and Piestun 2008; Piestun et al. 2008, 2009; Willemsen et al. 2010; Al-Zhgoul et al. 2013). Conceivably, the epigenetic regulators identified in this study could be involved in inducing the tolerance phenomena.

\section{Signaling pathways}

Two signal transduction cascades appear to be affected by heat stress: TGFß (Massague 1990; Lawrence 1996) and WNT (Clevers 2006). Five genes affecting the TGFß pathway were induced by heat stress, three of which, SMAD family member 6 (SMAD6), Endoglin (ENG), and TSC22 domain family, member 1 (TSC22D1), modulate signaling by direct interactions with components of the TGFß1 pathway. No changes were detected in the level of TGFß ligands as a function of heat stress. However, only ligands TGFB2 and TGFB3 have been identified in the chicken genome; consequently, our current analysis only quantifies expression of those two genes. The expression data for the TGF 3 receptors, TGFBR 1 and TGFBR2, are conflicting, with the level of TGFBR1 elevated (mean control, 45 RPKM; mean heat-treated, 65 RPKM), but TGFBR2 decreased by heat shock (mean control, 0.13 RPKM; mean heat-treated, undetected). One WNT ligand, WNT4, was increased threefold by heat treatment. An additional four other genes that were elevated by heat stress, Sp5 transcription factor (SP5), secreted frizzled-related protein 1 (SFRP1), dapper, antagonist of beta-catenin (DACT1), and sex determining region Y-box7 (SOX7), inhibit the WNT pathway, either by interfering with intracellular signaling or by blocking canonical WNT-mediated transcription activation (Cavallo et al. 1998; Uren et al. 2000; Takash et al. 2001; Zhang et al. 2006; Fujimura et al. 2007).

The increased expression of SMAD6, KLF6, and CTGF suggests that the TGFß pathway was activated during heat stress. This could be achieved, even in the absence of increased TGF $\beta$ ligand, by the increase in TGFßR 1 receptor expression. Elevated expression of this receptor might increase the sensitivity of cells to existing ligand levels. Increased signaling through the TGFß pathway inhibits apoptosis and improves cell survival during hyperthermia (Jia and Souchelnytskyi 2011). The WNT pathway typically promotes cellular growth. One WNT ligand, WNT4, was upregulated by hyperthermia, and this ligand functions as an antagonist of the canonical WNT signaling pathway. The remainder of the heat-induced genes affecting the WNT pathway typically inhibit WNT signaling. In addition, the chaperone encoded by the DNAJB6 gene has recently been shown to inhibit the canonical WNT signaling pathway (Mitra et al. 2010), and DNAJB6 was induced in the LMH cells by heat stress. The cumulative effect of heat stress on the TGFß and WNT pathways appears to be promoting cell survival during heat stress by inhibiting apoptosis (TGFß) and suppressing proliferation (WNT inhibition).

\section{DNA repair and replication}

DAVID functional enrichment analysis classified transcripts enriched in control cells into two groups: DNA replication and DNA repair. Characterizing this gene list with eGIFT iTerms supported the DAVID analysis (Table 6 and Fig. 3) and extended it to identify genes involved in chromosomal segregation and telomeric function. Some of these downregulated genes control the onset of $\mathrm{S}$ phase, including origin recognition complex, subunit 2 (ORC2), polo-like kinase 1 (PLK1), STE20-like kinase (SLK), and DBF4 homolog B (DBF4B), and decreased expression of these genes may slow the cell cycle. For example, the kinase PLK1 plays an important role in regulating mitotic entry, spindle formation, and cytokinesis (Lenart et al. 2007; Takaki et al. 2008) and PLK1 knockdown in non-transformed diploid cells prolongs $\mathrm{S}$ phase (Lei and Erikson 2008).

This study identified several genes affecting DNA repair and replication that are downregulated by heat stress. The ATR-Chk1 pathway plays a major role in repair of doublestranded breaks (Kampinga and Dikomey 2001; Krawczyk et al. 2011), and one of the several proteins that function in this pathway, Rad17 (Yan and Michael 2009a, 2009b; Tuul et al. 2013) is downregulated in LMH cells by heat stress. Several other proteins affecting DNA repair, DNA replication, spindle formation, chromatin structure, and cell cycle checkpoints were also downregulated by heat stress in the LMH cells (Fig. 3). Given that heat stress responses are frequently conserved across the evolutionary spectrum, these observations in a chicken liver cancer cell line may be relevant to hyperthermic treatment in oncology patients prior to radiation therapy. A major effect of radiation therapy is causing doublestranded breaks in DNA, thereby triggering apoptosis. Downregulating genes that repair such damage might play an important role in sensitizing tumors to radiation treatment (Kaur et al. 2011; Dewhirst and Chi 2013).

\section{Endoplasmic reticulum and Golgi}

eGIFT also identified the endoplasmic reticulum and Golgi as cellular compartments affected by genes downregulated by 
Table 6 iTERMS for genes significantly down-regulated by heat stress

\begin{tabular}{ll}
\hline iTERM & Gene symbols \\
\hline MITOSIS & RASGRF1, TNKS, KIF11, RCC2, RAD21, RAD50, PCNT, TUBA3E, TACC3, \\
& CDC14B, PAPD4, PLK1 TLK1, CDC20, KIF4A, DBF4B, CLIP1, ORC2L, \\
& CHAF1B, RAD17, SLK, NEDD9 \\
DNA DAMAGE & RASGRF1, FANCI, RAD21, ERCC3, RAD50, UIMC1 PLK1, PPP6C, DBF4B, \\
& TDG, TDP1, CHAF1B, RAD17, UHRF1, CCDC98 \\
MICROTUBULES & CKAP4, KIF11, RAD21, PCNT, TUBA3E, TACC3, GTSE1, PLK1, RANBP10, \\
& CDC20, KIF4A, CHFR, CLIP1, SLK \\
APOPTOSIS & MAP2K4, CKAP4, KIF11, FAF1, BAG1, HIP1R, IKBKB, PLK1, HIP1, SLK, NEDD9 \\
SPINDLE & KIF11, RCC2, RAD21, PCNT, TUBA3E, CDC14B, PLK1, EWSR1, KIF4A, DBF4B, CLIP1 \\
ACTIN CYTOSKELETON & TECR, CLIP1, TUBA3E, SLK, HIP1R, RHOC, MYO6 HIP1, MAGI2, DOCK1 \\
CHROMOSOME & TNKS, RAD21, RAD50, PCNT, ASPM, EWSR1, CDC20, TECR, ORC2L, RAD17 \\
DNA REPLICATION & RASGRF1, RAD21, TECR, DBF4B, ORC2L, TDP1, CHAF1B, UHRF1, RAD17, PIF1 \\
ENDOPLASMIC RETICULUM & LEPRE1, STIM1, RTN1, PTGES2, CYB5R4, PCSK7, MBTPS2, SCFD1, ALG1, ORAI1 \\
\hline
\end{tabular}

heat stress. Stromal interaction molecule 1 (STIM1) and ORAI calcium release-activated calcium modulator 1 (ORAI1) gene products are found in the endoplasmic reticulum and both modulate intracellular $\mathrm{Ca}^{2+}$ levels. STIM1 is a calcium sensor that activates plasma membrane ORAI1 when calcium levels are depleted in the ER (Putney 2007). This leads to replenishment of the ER calcium stores. Another downregulated gene, membrane-bound transcription factor peptidase, subunit 2 (MBTPS2), controls the ER stress response by functioning as a metalloprotease responsible for activating transcription factors, such as activating transcription factor 6 (ATF6), that control the unfolded protein

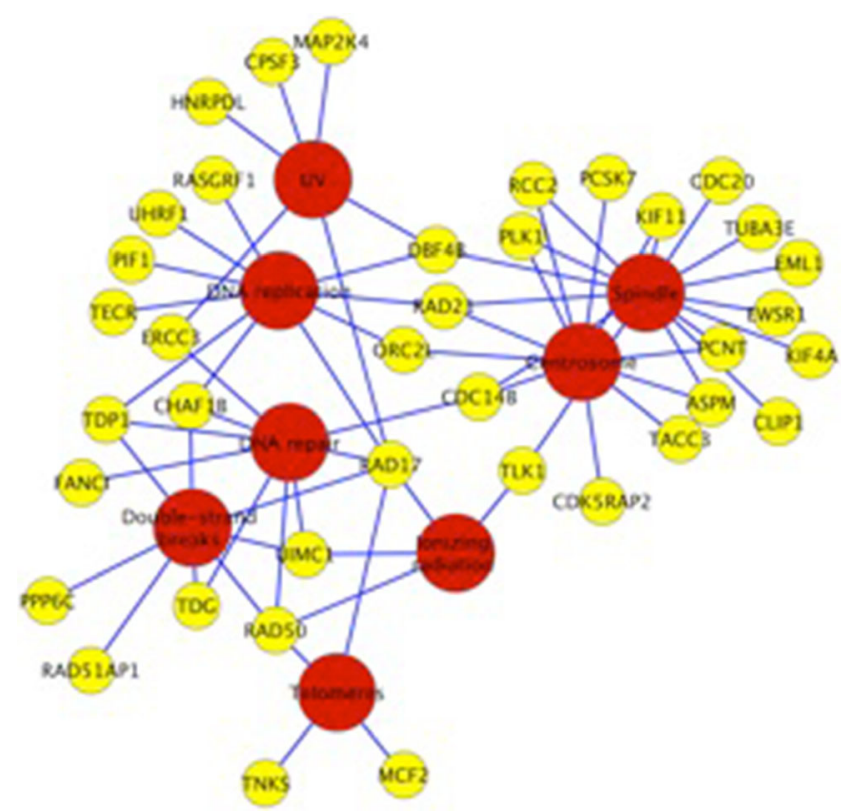

Fig. 3 Functional network of selected down-regulated genes. Genes down-regulated by heat stress and associated iTerms affecting DNA replication and repair processes. The yellow nodes correspond to gene products and the red nodes refer to iTerms associated with those gene products. response (Rawson et al. 1997; Haze et al. 1999). While ATF6 expression was detected in the LMH cells, its transcript level did not change in response to heat stress. Three downregulated genes function in protein modification including leucine-proline-enriched proteoglycan 1 (LEPRE1), asparagine-linked glycosylation 1, beta-1,4mannosyltransferase homolog (ALG1), and ST6 betagalactosamide alpha-2,6-sialyltranferase 1 (ST6GAL1). LEPRE1 and ALG1 function in the ER, with the former responsible for prolyl hydroxylase activity during collagen maturation (Vranka et al. 2004) while the latter carries out the first step in the production of lipid-linked oligosaccharides (Albright and Robbins 1990). ST6GAL1 is a sialyltransferase that functions in glycosylation in the Golgi (Weinstein et al. 1982, 1987). Finally, three downregulated gene products, sec1 family domain containing 1 (SCFD1), v-SNARE homolog (YKT6), and vacuolar protein sorting 8 homolog (VPS8), function in proper transport of cellular vesicles (Chen and Stevens 1996; Zhang and Hong 2001; Kosodo et al. 2002).

\section{Translation}

Two transcripts downregulated by heat stress, HSPA14 and MTOR associated protein, LST8 homolog (MLST8), have potential roles in controlling translation. HSPA14 encodes a protein that is associated with the ribosome and functions as a chaperone for nascent proteins, controlling their folding as they emerge from the ribosome. Why the expression of HSPA14 is downregulated in LMH cells during heat stress is uncertain. Potentially, reduction of HSPA14 could lower the rate of translation by slowing the emergence of properly folded proteins from the ribosome. This could contribute to reducing the protein synthesis burden during heat stress. MLST8 is a component of the mTORC1 complex, and one effect of mTORC1 activation is increased translation. 
Reduction of MLST8 levels would likely reduce overall protein synthesis by lowering the available pool of mTORC1. Taken together, downregulation of these two transcripts may play a direct role in the overall repression of protein synthesis seen during heat stress (Yamasaki and Anderson 2008; Shalgi et al. 2013)

\section{Correlation analysis}

Pearson correlation analysis was conducted on all differentially expressed genes and identified four networks of heatmodulated genes (Fig. 4). We limited this analysis to genes having connections to at least three other genes with correlation coefficients greater than 0.95 (Mansson et al. 2004). Two of the clusters contained downregulated genes (clusters 1 and 2) while the other two (clusters 3 and 4) contained upregulated genes. Highly correlated downregulated genes affected a variety of processes including DNA replication and repair, mRNA polyadenylation, pro-inflammatory responses, and glycosylation. The cluster of upregulated genes is predominantly comprised of genes encoding molecular chaperones along with some genes not previously associated with heat stress response. Finally, clusters 2, 3, and 4 are connected by several downregulated genes in cluster 2 that show strong negative correlation with several genes that are upregulated by heat stress.

A reasonable hypothesis is that individual clusters contain genes that are coordinately regulated by the same transcription factors. Given that HSFs form functional heterotrimers (Sandqvist et al. 2009), it is possible that different combinations of the HSFs have distinct transcription factor activities and could yield these different networks. Also, the strong negative correlations between up regulated and downregulated genes (i.e., HSPA4 and MLST8) in clusters 2, 3, and 4 suggest a mechanism in which the same transcription factor is positively regulating one set of genes (clusters 3 and 4), while negatively regulating another set (cluster 2 ). Future work can use overexpression and knockdown approaches to evaluate the impact of changing the levels of different transcription factors on these networks.

It is possible that heat responsive genes may function in setting body temperature. The Animal Quantitative Trait Loci (QTL) Database (Hu et al. 2013)(Release 24, Aug 25, 2014) identifies QTLs associated with body temperature mapped to five chicken chromosomes (chromosomes 2, 3, 4, 6, and 23). Inspecting the chromosomal locations of the heat stress responsive genes defined in this study (Table 7) provides a total of 25 genes located within the QTL boundaries on chromosomes 2, 3, 4, and 6 . These 25 can be considered candidate genes for regulating body temperature.

Overall, this work has identified 812 genes whose transcription is modified by heat stress. Based on numerous previous studies, many of the genes identified in the current study were predicted to be responsive to heat stress (Lindquist and Craig 1988; Akerfelt et al. 2010). However, several of the genes either upregulated or downregulated by heat challenge in our studies have not, to our knowledge, been previously identified as heat responsive. Multiple biological processes were affected by the responsive genes including translation, transcription, chromatin modification, DNA repair, and DNA synthesis. In addition, two signaling pathways were modulated by heat stress: TGFß and WNT. The heat responsive genes affecting the TGFß pathway indicate activation of this pathway, while the WNT pathway appears to be inactivated. Current studies in our laboratory are using RNA-seq to identify heat responsive genes following hyperthermic treatment
Fig. 4 Pearson Correlation Network Networks containing significantly correlated gene pairs (Pearson correlation coefficient> 0.95 ) that were modulated either up or down by heat stress. Nodes in cluster 1 (green) or cluster 2 (purple) were down-regulated in heat challenge while nodes in cluster 3 (gray) or cluster 4 (brown) were up-regulated. Red edges indicate positive correlation between gene pairs while blue edges indicate negative correlation.

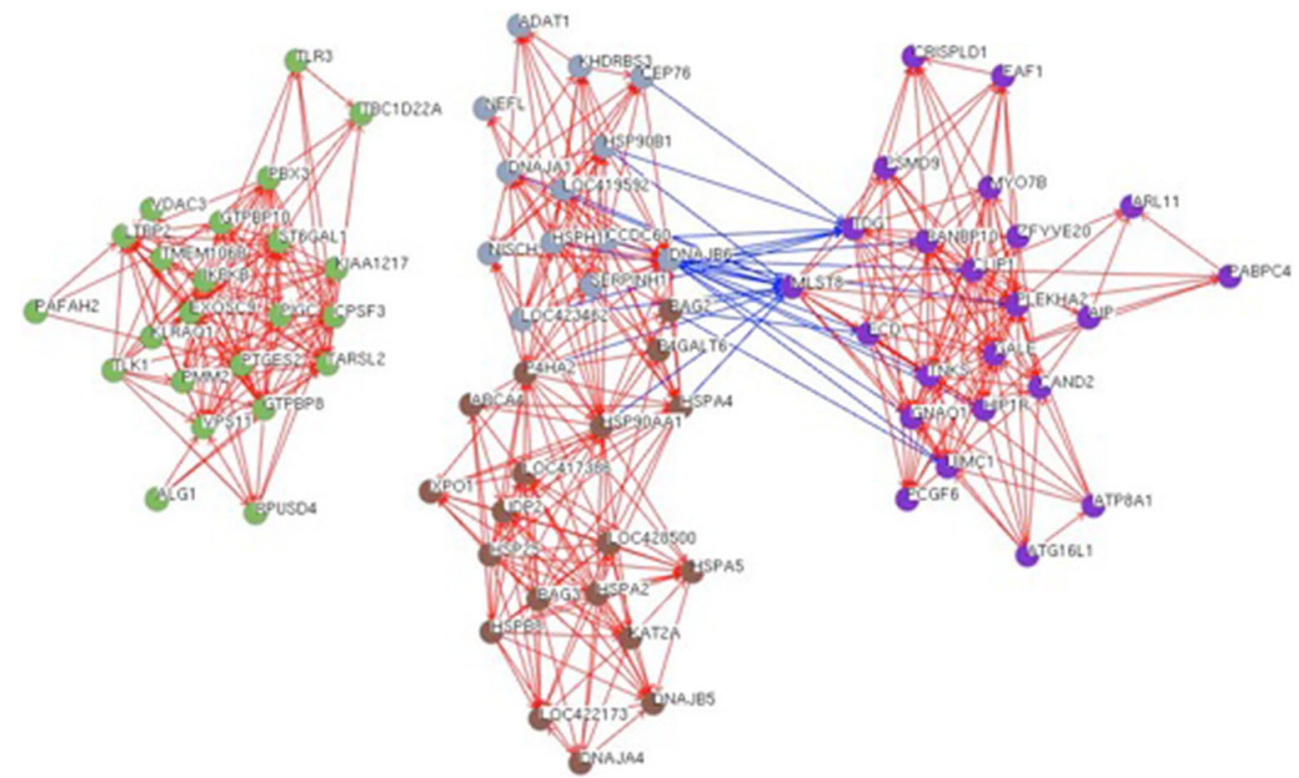


Table 7 Heat Responsive genes mapping to QTL locations that regulate body temperature. QTL locations were obtained from the Animal QTL Database

\begin{tabular}{|c|c|c|c|c|c|}
\hline GENE & CHROMOSOME & Start & Finish & Ave. Control RPKM & Ave. Heat Stress RPKM \\
\hline NSUN2 & 2 & $82,300,000$ & $87,200,000$ & 93.5 & 49.4 \\
\hline MOCOS & 2 & $82,300,000$ & $87,200,000$ & 2.01 & 1.08 \\
\hline PDE10A & 3 & $35,500,000$ & $51,300,000$ & 12.5 & 28.5 \\
\hline PNLDC1 & 3 & $35,500,000$ & $51,300,000$ & 0.09 & 0.24 \\
\hline SHPRH & 3 & $35,500,000$ & $51,300,000$ & 1.65 & 0.87 \\
\hline UST & 3 & $35,500,000$ & $51,300,000$ & 3.80 & 7.25 \\
\hline EXOSC9 & 4 & $50,900,000$ & $88,400,000$ & 35.7 & 12.6 \\
\hline SYNPO2 & 4 & $50,900,000$ & $88,400,000$ & 0.35 & 0.13 \\
\hline METTL14 & 4 & $50,900,000$ & $88,400,000$ & 24.4 & 13.5 \\
\hline TLR3 & 4 & $50,900,000$ & $88,400,000$ & 3.28 & 1.64 \\
\hline SLC7A2 & 4 & $50,900,000$ & $88,400,000$ & 0.89 & 0.34 \\
\hline KCTD8 & 4 & $50,900,000$ & $88,400,000$ & 4.00 & 1.25 \\
\hline ATP8A1 & 4 & $50,900,000$ & $88,400,000$ & 3.66 & 1.80 \\
\hline RBM47 & 4 & $50,900,000$ & $88,400,000$ & 28.9 & 16.5 \\
\hline CHRNA9 & 4 & $50,900,000$ & $88,400,000$ & 0.33 & 0.08 \\
\hline PCDH7 & 4 & $50,900,000$ & $88,400,000$ & 0.88 & 0.44 \\
\hline ANAPC4 & 4 & $50,900,000$ & $88,400,000$ & 7.65 & 4.23 \\
\hline CCDC149 & 4 & $50,900,000$ & $88,400,000$ & 4.84 & 2.11 \\
\hline TBC1D14 & 4 & $50,900,000$ & $88,400,000$ & 14.3 & 7.18 \\
\hline AFAP1 & 4 & $50,900,000$ & $88,400,000$ & 3.70 & 8.21 \\
\hline TACC3 & 4 & $50,900,000$ & $88,400,000$ & 78.7 & 29.6 \\
\hline LOC423899 & 6 & $4,500,000$ & $33,800,000$ & 0.26 & 0.07 \\
\hline BAG3 & 6 & $4,500,000$ & $33,800,000$ & 33.8 & 806.0 \\
\hline LHPP & 6 & $4,500,000$ & $33,800,000$ & 11.1 & 6.15 \\
\hline DOCK1 & 6 & $4,500,000$ & $33,800,000$ & 6.11 & 2.65 \\
\hline
\end{tabular}

of chickens with the goal of providing a more complete cata$\log$ of heat responsive genes.

\section{Broader implications}

We have examined the transcriptome response of the chicken LMH hepatocarcinoma cell line to hyperthermia using RNA-seq and identified biological processes and pathways impacted by heat stress. It is important to recognize the nature of the cells studied; the LMH cell line was obtained from a male chicken fed diethylnitrosamine, a potent inducer of liver cancer (Kawaguchi et al. 1987). The line was derived in 1987 and has adapted to long-term growth in cell culture. When comparing our results with analyses of response to heat stress in other cell lines or tissues, both similarities and differences are noted. Among the major similarities across cell lines from different species are increases in transcripts encoding chaperones and cochaperones that play important roles in maintaining homeostasis during heat stress (Lindquist and Craig 1988; Murray et al. 2004). Responses in these functional groups of genes are also noted in the livers of chickens (Schmidt, unpublished), ducks (Zeng et al. 2014), and other animals (Flanagan et al. 1995). Another common response to heat stress are changes in gene expression consistent with cell cycle arrest (Sonna et al. 2002) although this is variable. For example, the human fibroblasts exhibit cell cycle arrest during hyperthermia while human Hela and K-562 cancer cell lines do not (Murray et al. 2004). Upregulation of the TGFß pathway through different mechanisms has been noted in other cell culture systems (Jia and Souchelnytskyi 2011; Li et al. 2015) and in scrotal tissues (Cai et al. 2011) during heat stress. In reviewing this information, it becomes apparent that not all cell lines or tissues respond to heat stress in exactly the same fashion. The total response likely depends on a combination of variables including the genetic composition of the target species, the genomic changes that have occurred due to past environmental stresses, and the nature of the current heat challenge (Sonna et al. 2002). An important future goal will be to relate heat stress transcriptome responses at the cellular and organismal level to these different variables. 
Acknowledgments This project was supported by Agriculture and Food Research Initiative Competitive Grant 2011-67003-30228 from the USDA National Institute of Food and Agriculture. Activities at Iowa State University were also supported by the College of Agriculture and Life Sciences, State of Iowa and Hatch funding.

\section{Appendix}

The data sets supporting the results of this article are included within the article and its additional files

Open Access This article is distributed under the terms of the Creative Commons Attribution 4.0 International License (http:// creativecommons.org/licenses/by/4.0/), which permits unrestricted use, distribution, and reproduction in any medium, provided you give appropriate credit to the original author(s) and the source, provide a link to the Creative Commons license, and indicate if changes were made.

\section{References}

Akerfelt M, Morimoto RI, Sistonen L (2010) Heat shock factors: integrators of cell stress, development and lifespan. Nat Rev Mol Cell Biol 11(8):545-555

Al-Zhgoul MB, Dalab AE, Ababneh MM, Jawasreh KI, Al Busadah KA, Ismail ZB (2013) Thermal manipulation during chicken embryogenesis results in enhanced Hsp70 gene expression and the acquisition of thermotolerance. Res Vet Sci 95(2):502-507

Albright CF, Robbins RW (1990) The sequence and transcript heterogeneity of the yeast gene ALG1, an essential mannosyltransferase involved in N-glycosylation. J Biol Chem 265(12):7042-7049

Alfieri C, Gambetta MC, Matos R, Glatt S, Sehr P, Fraterman S, Wilm M, Muller J, Muller CW (2013) Structural basis for targeting the chromatin repressor Sfmbt to Polycomb response elements. Genes Dev 27(21):2367-2379

Brigstock DR (2002) Regulation of angiogenesis and endothelial cell function by connective tissue growth factor (CTGF) and cysteinerich 61 (CYR61). Angiogenesis 5(3):153-165

Cai H, Ren Y, Li XX, Yang JL, Zhang CP, Chen M, Fan CH, Hu XQ, Hu ZY, Gao F, Liu YX (2011) Scrotal heat stress causes a transient alteration in tight junctions and induction of TGF-beta expression. Int J Androl 34(4):352-362

Cai L, Fritz D, Stefanovic L, Stefanovic B (2010a) Binding of LARP6 to the conserved 5' stem-loop regulates translation of mRNAs encoding type I collagen. J Mol Biol 395(2):309-326

Cai L, Fritz D, Stefanovic L, Stefanovic B (2010b) Nonmuscle myosindependent synthesis of type I collagen. J Mol Biol 401(4):564-578

Camarena L, Bruno V, Euskirchen G, Poggio S, Snyder M (2010) Molecular mechanisms of ethanol-induced pathogenesis revealed by RNA-sequencing. PLoS Pathog 6(4), e1000834

Cates GA, Nandan D, Brickenden AM, Sanwal BD (1987) Differentiation defective mutants of skeletal myoblasts altered in a gelatin-binding glycoprotein. Biochem Cell Biol 65(9):767-775

Cavallo RA, Cox RT, Moline MM, Roose J, Polevoy GA, Clevers H, Peifer M, Bejsovec A (1998) Drosophila Tcf and Groucho interact to repress Wingless signalling activity. Nature 395(6702):604-608

Chang B, Chen Y, Zhao Y, Bruick RK (2007) JMJD6 is a histone arginine demethylase. Science 318(5849):444-447

Chen YJ, Stevens TH (1996) The VPS8 gene is required for localization and trafficking of the CPY sorting receptor in Saccharomyces cerevisiae. Eur J Cell Biol 70(4):289-297
Clevers H (2006) Wnt/beta-catenin signaling in development and disease. Cell 127(3):469-480

Core LJ, Waterfall JJ, Lis JT (2008) Nascent RNA sequencing reveals widespread pausing and divergent initiation at human promoters. Science 322(5909): 1845-1848

da Huang W, Sherman BT, Lempicki RA (2009a) Bioinformatics enrichment tools: paths toward the comprehensive functional analysis of large gene lists. Nucleic Acids Res 37(1):1-13

da Huang W, Sherman BT, Lempicki RA (2009b) Systematic and integrative analysis of large gene lists using DAVID bioinformatics resources. Nat Protoc 4(1):44-57

de Bruin A, Maiti B, Jakoi L, Timmers C, Buerki R, Leone G (2003) Identification and characterization of E2F7, a novel mammalian E2F family member capable of blocking cellular proliferation. J Biol Chem 278(43):42041-42049

Dewhirst MW, Chi JT (2013) Understanding the tumor microenvironment and radioresistance by combining functional imaging with global gene expression. Semin Radiat Oncol 23(4):296-305

Duncan MR, Frazier KS, Abramson S, Williams S, Klapper H, Huang X, Grotendorst GR (1999) Connective tissue growth factor mediates transforming growth factor beta-induced collagen synthesis: downregulation by cAMP. FASEB J 13(13):1774-1786

Feder ME, Hofmann GE (1999) Heat-shock proteins, molecular chaperones, and the stress response: evolutionary and ecological physiology. Annu Rev Physiol 61:243-282

Feng L, Liu H, Liu Y, Lu Z, Guo G, Guo S, Zheng H, Gao Y, Cheng S, Wang J, Zhang K, Zhang Y (2010) Power of deep sequencing and agilent microarray for gene expression profiling study. Mol Biotechnol 45(2):101-110

Flanagan SW, Ryan AJ, Gisolfi CV, Moseley PL (1995) Tissue-specific HSP70 response in animals undergoing heat stress. Am J Physiol 268(1 Pt 2):R28-32

Fujimura N, Vacik T, Machon O, Vlcek C, Scalabrin S, Speth M, Diep D, Krauss S, Kozmik Z (2007) Wnt-mediated down-regulation of Sp1 target genes by a transcriptional repressor Sp5. J Biol Chem 282(2): 1225-1237

Gabis KK, Gildemeister OS, Pepe JA, Lambrecht RW, Bonkovsky HL (1996) Induction of heme oxygenase-1 in LMH cells. Comparison of LMH cells to primary cultures of chick embryo liver cells. Biochim Biophys Acta 1290(1):113-120

Hashimoto H, Horton JR, Zhang X, Cheng X (2009) UHRF1, a modular multi-domain protein, regulates replication-coupled crosstalk between DNA methylation and histone modifications. Epigenetics 4(1):8-14

Haze K, Yoshida H, Yanagi H, Yura T, Mori K (1999) Mammalian transcription factor ATF6 is synthesized as a transmembrane protein and activated by proteolysis in response to endoplasmic reticulum stress. Mol Biol Cell 10(11):3787-3799

Hightower LE (1991) Heat shock, stress proteins, chaperones, and proteotoxicity. Cell 66(2):191-197

Horvath GC, Kistler WS, Kistler MK (2004) RFX2 is a potential transcriptional regulatory factor for histone $\mathrm{H} 1 \mathrm{t}$ and other genes expressed during the meiotic phase of spermatogenesis. Biol Reprod 71(5):1551-1559

Hu ZL, Park CA, Wu XL, Reecy JM (2013) Animal QTLdb: an improved database tool for livestock animal QTL/association data dissemination in the post-genome era. Nucleic Acids Res 41(Database issue): D871-879

International Chicken Genome Sequencing, C (2004) Sequence and comparative analysis of the chicken genome provide unique perspectives on vertebrate evolution. Nature 432(7018):695-716

Ishikawa H, Ma Z, Barber GN (2009) STING regulates intracellular DNA-mediated, type I interferon-dependent innate immunity. Nature 461(7265):788-792 
Islam A, Deuster PA, Devaney JM, Ghimbovschi S, Chen Y (2013) An exploration of heat tolerance in mice utilizing mRNA and microRNA expression analysis. PLoS One 8(8), e72258

Jia M, Souchelnytskyi S (2011) Proteome profiling of heat shock of human primary breast epithelial cells, a dataset report. Cell Stress Chaperones 16(4):459-467

Jin C, Kato K, Chimura T, Yamasaki T, Nakade K, Murata T, Li H, Pan J, Zhao M, Sun K, Chiu R, Ito T, Nagata K, Horikoshi M, Yokoyama KK (2006) Regulation of histone acetylation and nucleosome assembly by transcription factor JDP2. Nat Struct Mol Biol 13(4): 331-338

Kampinga HH, Dikomey E (2001) Hyperthermic radiosensitization: mode of action and clinical relevance. Int J Radiat Biol 77(4):399_ 408

Kaur P, Hurwitz MD, Krishnan S, Asea A (2011) Combined hyperthermia and radiotherapy for the treatment of cancer. Cancers (Basel) 3(4):3799-3823

Kawaguchi T, Nomura K, Hirayama Y, Kitagawa T (1987) Establishment and characterization of a chicken hepatocellular carcinoma cell line, LMH. Cancer Res 47(16):4460-4464

Kester HA, Blanchetot C, den Hertog J, van der Saag PT, van der Burg B (1999) Transforming growth factor-beta-stimulated clone-22 is a member of a family of leucine zipper proteins that can homo- and heterodimerize and has transcriptional repressor activity. J Biol Chem 274(39):27439-27447

Kosodo Y, Noda Y, Adachi H, Yoda K (2002) Binding of Sly1 to Sed5 enhances formation of the yeast early Golgi SNARE complex. J Cell Sci 115(Pt 18):3683-3691

Krawczyk PM, Eppink B, Essers J, Stap J, Rodermond H, Odijk H, Zelensky A, van Bree C, Stalpers LJ, Buist MR, Soullie T, Rens J, Verhagen HJ, O'Connor MJ, Franken NA, Ten Hagen TL, Kanaar R, Aten JA (2011) Mild hyperthermia inhibits homologous recombination, induces BRCA2 degradation, and sensitizes cancer cells to poly (ADP-ribose) polymerase-1 inhibition. Proc Natl Acad Sci U S A 108(24):9851-9856

Kregel KC (2002) Heat shock proteins: modifying factors in physiological stress responses and acquired thermotolerance. J Appl Physiol (1985) 92(5):2177-2186

Kristiansson E, Osterlund T, Gunnarsson L, Arne G, Larsson DG, Nerman O (2013) A novel method for cross-species gene expression analysis. BMC Bioinformatics 14:70

Kurkinen M, Taylor A, Garrels JI, Hogan BL (1984) Cell surfaceassociated proteins which bind native type IV collagen or gelatin. J Biol Chem 259(9):5915-5922

Lawrence DA (1996) Transforming growth factor-beta: a general review. Eur Cytokine Netw 7(3):363-374

Leco KJ, Waterhouse P, Sanchez OH, Gowing KL, Poole AR, Wakeham A, Mak TW, Khokha R (2001) Spontaneous air space enlargement in the lungs of mice lacking tissue inhibitor of metalloproteinases-3 (TIMP-3). J Clin Invest 108(6):817-829

Lei M, Erikson RL (2008) Plk1 depletion in nontransformed diploid cells activates the DNA-damage checkpoint. Oncogene 27(28):39353943

Lenart P, Petronczki M, Steegmaier M, Di Fiore B, Lipp JJ, Hoffmann M, Rettig WJ, Kraut N, Peters JM (2007) The small-molecule inhibitor BI 2536 reveals novel insights into mitotic roles of polo-like kinase 1. Curr Biol 17(4):304-315

Li C, Wang X, Wang G, Li N, Wu C (2011) Expression analysis of global gene response to chronic heat exposure in broiler chickens (Gallus gallus) reveals new reactive genes. Poult Sci 90(5):1028-1036

Li L, Sun Y, Wu J, Li X, Luo M, Wang G (2015) The global effect of heat on gene expression in cultured bovine mammary epithelial cells. Cell Stress Chaperones 20(2):381-389

Lindquist S, Craig EA (1988) The heat-shock proteins. Annu Rev Genet 22:631-677
Liu Y, Wang J, Yi Y, Zhang H, Liu J, Liu M, Yuan C, Tang D, Benjamin IJ, Xiao X (2006) Induction of KLF4 in response to heat stress. Cell Stress Chaperones 11(4):379-389

Mansson R, Tsapogas P, Akerlund M, Lagergren A, Gisler R, Sigvardsson M (2004) Pearson correlation analysis of microarray data allows for the identification of genetic targets for early B-cell factor. J Biol Chem 279(17):17905-17913

Massague J (1990) The transforming growth factor-beta family. Annu Rev Cell Biol 6:597-641

Mitra A, Menezes ME, Shevde LA, Samant RS (2010) DNAJB6 induces degradation of beta-catenin and causes partial reversal of mesenchymal phenotype. J Biol Chem 285(32):24686-24694

Mortazavi A, Williams BA, McCue K, Schaeffer L, Wold B (2008) Mapping and quantifying mammalian transcriptomes by RNASeq. Nat Methods 5(7):621-628

Moseley PL (1997) Heat shock proteins and heat adaptation of the whole organism. J Appl Physiol (1985) 83(5):1413-1417

Murray JI, Whitfield ML, Trinklein ND, Myers RM, Brown PO, Botstein D (2004) Diverse and specific gene expression responses to stresses in cultured human cells. Mol Biol Cell 15(5):2361-2374

Nabatiyan A, Krude T (2004) Silencing of chromatin assembly factor 1 in human cells leads to cell death and loss of chromatin assembly during DNA synthesis. Mol Cell Biol 24(7):2853-2862

Nagalakshmi U, Wang Z, Waern K, Shou C, Raha D, Gerstein M, Snyder M (2008) The transcriptional landscape of the yeast genome defined by RNA sequencing. Science 320(5881):1344-1349

Nagata K, Saga S, Yamada KM (1986) A major collagen-binding protein of chick embryo fibroblasts is a novel heat shock protein. J Cell Biol 103(1):223-229

Nagy Z, Tora L (2007) Distinct GCN5/PCAF-containing complexes function as co-activators and are involved in transcription factor and global histone acetylation. Oncogene 26(37):5341-5357

Olaso E, Arteta B, Benedicto A, Crende O, Friedman SL (2011) Loss of discoidin domain receptor 2 promotes hepatic fibrosis after chronic carbon tetrachloride through altered paracrine interactions between hepatic stellate cells and liver-associated macrophages. Am J Pathol 179(6):2894-2904

Pavani SR, Piestun R (2008) Three dimensional tracking of fluorescent microparticles using a photon-limited double-helix response system. Opt Express 16(26):22048-22057

Pavloff N, Staskus PW, Kishnani NS, Hawkes SP (1992) A new inhibitor of metalloproteinases from chicken: ChIMP-3. A third member of the TIMP family. J Biol Chem 267(24):17321-17326

Piestun Y, Harel M, Barak M, Yahav S, Halevy O (2009) Thermal manipulations in late-term chick embryos have immediate and longer term effects on myoblast proliferation and skeletal muscle hypertrophy. J Appl Physiol (1985) 106(1):233-240

Piestun Y, Shinder D, Ruzal M, Halevy O, Brake J, Yahav S (2008) Thermal manipulations during broiler embryogenesis: effect on the acquisition of thermotolerance. Poult Sci 87(8):1516-1525

Putney JW Jr (2007) New molecular players in capacitative Ca2+ entry. J Cell Sci 120(Pt 12):1959-1965

Rajasekhar VK, Begemann M (2007) Concise review: roles of polycomb group proteins in development and disease: a stem cell perspective. Stem Cells 25(10):2498-2510

Rawson RB, Zelenski NG, Nijhawan D, Ye J, Sakai J, Hasan MT, Chang TY, Brown MS, Goldstein JL (1997) Complementation cloning of S2P, a gene encoding a putative metalloprotease required for intramembrane cleavage of SREBPs. Mol Cell 1(1):47-57

Sandqvist A, Bjork JK, Akerfelt M, Chitikova Z, Grichine A, Vourc'h C, Jolly C, Salminen TA, Nymalm Y, Sistonen L (2009) Heterotrimerization of heat-shock factors 1 and 2 provides a transcriptional switch in response to distinct stimuli. Mol Biol Cell 20(5):1340-1347 
Shalgi R, Hurt JA, Krykbaeva I, Taipale M, Lindquist S, Burge CB (2013) Widespread regulation of translation by elongation pausing in heat shock. Mol Cell 49(3):439-452

Sjostrom M, Stenstrom K, Eneling K, Zwiller J, Katz AI, Takemori H, Bertorello AM (2007) SIK1 is part of a cell sodium-sensing network that regulates active sodium transport through a calcium-dependent process. Proc Natl Acad Sci U S A 104(43):16922-16927

Smith S, Bernatchez L, Beheregaray LB (2013) RNA-seq analysis reveals extensive transcriptional plasticity to temperature stress in a freshwater fish species. BMC Genomics 14:375

Sonna LA, Fujita J, Gaffin SL, Lilly CM (2002) Invited review: effects of heat and cold stress on mammalian gene expression. J Appl Physiol (1985) 92(4):1725-1742

Sun R, Chen X, Yang VW (2001) Intestinal-enriched Kruppel-like factor (Kruppel-like factor 5) is a positive regulator of cellular proliferation. J Biol Chem 276(10):6897-6900

Szustakowski JD, Kosinski PA, Marrese CA, Lee JH, Elliman SJ, Nirmala N, Kemp DM (2007) Dynamic resolution of functionally related gene sets in response to acute heat stress. BMC Mol Biol 8:46

Takaki T, Trenz K, Costanzo V, Petronczki M (2008) Polo-like kinase 1 reaches beyond mitosis-cytokinesis, DNA damage response, and development. Curr Opin Cell Biol 20(6):650-660

Takash W, Canizares J, Bonneaud N, Poulat F, Mattei MG, Jay P, Berta P (2001) SOX7 transcription factor: sequence, chromosomal localisation, expression, transactivation and interference with Wnt signalling. Nucleic Acids Res 29(21):4274-4283

Tudor CO, Schmidt CJ, Vijay-Shanker K (2010) eGIFT: mining gene information from the literature. BMC Bioinformatics 11:418

Tuul M, Kitao H, Iimori M, Matsuoka K, Kiyonari S, Saeki H, Oki E, Morita M, Maehara Y (2013) Rad9, Rad17, TopBP1 and claspin play essential roles in heat-induced activation of ATR kinase and heat tolerance. PLoS One 8(2), e55361

Uren A, Reichsman F, Anest V, Taylor WG, Muraiso K, Bottaro DP, Cumberledge S, Rubin JS (2000) Secreted frizzled-related protein1 binds directly to Wingless and is a biphasic modulator of Wnt signaling. J Biol Chem 275(6):4374-4382

Vranka JA, Sakai LY, Bachinger HP (2004) Prolyl 3-hydroxylase 1, enzyme characterization and identification of a novel family of enzymes. J Biol Chem 279(22):23615-23621

Wang J, Bhutani M, Pathak AK, Lang W, Ren H, Jelinek J, He R, Shen L, Issa JP, Mao L (2007) Delta DNMT3B variants regulate DNA methylation in a promoter-specific manner. Cancer Res 67(22):1064710652

Wang S, Edens FW (1998) Heat conditioning induces heat shock proteins in broiler chickens and turkey poults. Poult Sci 77(11):1636-1645
Wang SH, Cheng CY, Tang PC, Chen CF, Chen HH, Lee YP, Huang SY (2013) Differential gene expressions in testes of L2 strain Taiwan country chicken in response to acute heat stress. Theriogenology 79(2):374-382, e371-377

Wang Z, Takemori H, Halder SK, Nonaka Y, Okamoto M (1999) Cloning of a novel kinase (SIK) of the SNF1/AMPK family from high salt diet-treated rat adrenal. FEBS Lett 453(1-2):135-139

Weinstein J, de Souza-e-Silva U, Paulson JC (1982) Sialylation of glycoprotein oligosaccharides N-linked to asparagine. Enzymatic characterization of a Gal beta 1 to 3(4)GlcNAc alpha 2 to 3 sialyltransferase and a Gal beta 1 to $4 \mathrm{GlcNAc}$ alpha 2 to 6 sialyltransferase from rat liver. J Biol Chem 257(22):13845-13853

Weinstein J, Lee EU, McEntee K, Lai PH, Paulson JC (1987) Primary structure of beta-galactoside alpha 2,6-sialyltransferase. Conversion of membrane-bound enzyme to soluble forms by cleavage of the NH2-terminal signal anchor. J Biol Chem 262(36):17735-17743

Willemsen H, Kamers B, Dahlke F, Han H, Song Z, Ansari Pirsaraei Z, Tona K, Decuypere E, Everaert N (2010) High- and lowtemperature manipulation during late incubation: effects on embryonic development, the hatching process, and metabolism in broilers. Poult Sci 89(12):2678-2690

Wolfe SA, Wilkerson DC, Prado S, Grimes SR (2004) Regulatory factor X2 (RFX2) binds to the H1t/TE1 promoter element and activates transcription of the testis-specific histone H1t gene. J Cell Biochem 91(2):375-383

Yamasaki S, Anderson P (2008) Reprogramming mRNA translation during stress. Curr Opin Cell Biol 20(2):222-226

Yan S, Michael WM (2009a) TopBP1 and DNA polymerase alphamediated recruitment of the 9-1-1 complex to stalled replication forks: implications for a replication restart-based mechanism for ATR checkpoint activation. Cell Cycle 8(18):2877-2884

Yan S, Michael WM (2009b) TopBP1 and DNA polymerase-alpha directly recruit the 9-1-1 complex to stalled DNA replication forks. J Cell Biol 184(6):793-804

Zeng T, Li JJ, Wang DQ, Li GQ, Wang GL, Lu LZ (2014) Effects of heat stress on antioxidant defense system, inflammatory injury, and heat shock proteins of Muscovy and Pekin ducks: evidence for differential thermal sensitivities. Cell Stress Chaperones 19(6):895-901

Zhang L, Gao X, Wen J, Ning Y, Chen YG (2006) Dapper 1 antagonizes Wnt signaling by promoting dishevelled degradation. J Biol Chem 281(13):8607-8612

Zhang T, Hong W (2001) Ykt6 forms a SNARE complex with syntaxin 5, GS28, and Bet1 and participates in a late stage in endoplasmic reticulum-Golgi transport. J Biol Chem 276(29):27480-27487 